\title{
Modelización de la distribución potencial del género Polylepis en Colombia y consideraciones para su conservación
}

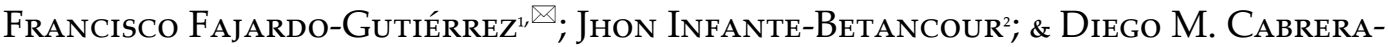 \\ AMAYA ${ }^{1 / 2}$ \\ ${ }^{1}$ Jardín Botánico de Bogotá José Celestino Mutis, Bogotá, Colombia. ${ }^{2}$ Yoluka ONG, Fundación de investigación en \\ biodiversidad y conservación, Bogotá, Colombia.
}

\begin{abstract}
Resumen. En Colombia, el género Polylepis está representado por P. quadrijuga, P. sericea y P. incana. La modelización de la distribución geográfica potencial permite identificar áreas relevantes para conservar especies focales. Los objetivos de este trabajo fueron establecer las áreas de distribución potencial de las tres especies e identificar oportunidades de conservación en áreas protegidas a escala nacional, regional y local. Para esto, se recopilaron 223 registros biológicos (GBIF y SIB Colombia) que, junto con 23 variables ambientales, fueron los insumos para modelizar la distribución potencial en MaxEnt 3.3.3K. Como oportunidades de conservación, se cuantificó la presencia de reservas del SINAP-Colombia en las áreas de distribución potencial de las especies. Las variables que más contribuyen en los modelos son altitud, temperatura estacional y rango de temperatura media mensual, entre otras. $P$. sericea presentó el área de distribución potencial más amplia, con $23243.5 \mathrm{~km}^{2} \mathrm{en}$ las tres cordilleras; le siguieron $P$. quadrijuga, con $6264.2 \mathrm{~km}^{2}$ en la Cordillera Oriental y P. incana, con $1772.8 \mathrm{~km}^{2}$ en la Cordillera Central y la frontera con Ecuador. Una parte de dichas distribuciones potenciales se encuentra en, al menos, un tipo de área protegida. P. incana tiene $59.5 \%$ del área de distribución potencial dentro de alguna figura de protección, P. quadrijuga presenta $44.8 \%$ de área potencial de distribución ubicada dentro de cualquier figura de protección y $P$. sericea, al tener una mayor superficie potencial a partir de la modelización, posee un porcentaje más bajo de representatividad (33.7\%) en áreas protegidas del SINAP. Finalmente, a partir del análisis de amenazas y oportunidades se proponen áreas clave para la conservación de Polylepis y zonas para la búsqueda de posibles poblaciones no reportadas.
\end{abstract}

[Palabras clave: páramo, Andes, áreas protegidas, bosque, MaxEnt, P. quadrijuga, P. sericea, P. incana]

Aвstract. Modelling the potential distribution of the genus Polylepis occurring in Colombia with conservation considerations. The genus Polylepis in Colombia is represented by P. quadrijuga, $P$. sericea and $P$. incana. The modeling of the potential geographic distribution allows identifying areas of importance for the conservation of focal species. The objectives of this work were to establish the potential distribution areas of the three species and identify conservation opportunities in protected areas at national, regional and local levels. For this, 223 biological records were compiled (mainly from GBIF and SIB Colombia), which, together with 23 environmental variables, were the inputs for the modeling of the potential distribution in MaxEnt 3.3.3K. As conservation opportunities, the presence of reserves of SINAP-Colombia in the areas of potential distribution of the species was quantified. The variables that contribute most in the models are altitude, seasonal temperature and monthly average temperature range, among others. $P$. sericea showed the largest potential distribution area, with $23243.5 \mathrm{~km}^{2}$ in the three mountain ranges, followed by P. quadrijuga, with $6264.2 \mathrm{~km}^{2}$ in the Eastern Cordillera, and P. incana, with $1772.8 \mathrm{~km}^{2}$ in the Central Mountain Range and the border with Ecuador. A part of said potential distributions are found in, at least, one type of protected area. $P$. incana has $59.5 \%$ of the potential distribution area within some protection figure, followed by P. quadrijuga, with $44.8 \%$ potential distribution area located within any protection figure, and P. sericea, which, having a larger area potential result of the modeling, showed a lower percentage (33.7\%) of representativeness in protected areas of SINAP. Finally, from the analysis of threats and opportunities, key areas for the conservation of Polylepis and zones for the search of possible non-reported populations are proposed.

[Keywords: páramo, Andes, protected areas, forest, MaxEnt, P. quadrijuga, P. sericea, P. incana]

Editora asociada: Erica Cuyckens

fajardo.pacho@gmail.com; ffajardo@jbb.gov.co
Recibido: 2 de mayo de 2017

Aceptado: 7 de diciembre de 2017 


\section{INTRODUCCIÓN}

En toda Sudamérica, los bosques de Polylepis están sujetos a una gran fragmentación y degradación del hábitat (Tarifa and Yensen 2001; Rangel-Ch and Arellano-P. 2010), principalmente por el uso indiscriminado para madera y leña (Kessler and Driesch 1993) y a la expansión de la frontera agrícola y ganadera (Fandiño and Caro-Sabogal 2009; Rangel-Ch and Arellano-P. 2010). Este género está representado en Colombia por tres especies, de acuerdo con el Catálogo de Plantas y Líquenes de Colombia (Pérez 2017): Polylepis incana Kunth, Polylepis quadrijuga Bitter y Polylepis sericea Wedd. De éstas, P. sericea presenta una distribución amplia, $P$. incana tiene distribución restringida y $P$. quadrijuga es endémica del país. En Colombia, el género Polylepis se encuentra en los páramos entre 2800 y 4300 m s. n. m., lo que los convierte en una de las especies leñosas de hábito arbóreo que alcanza mayores altitudes en los Andes (Pérez 2017).

El conocimiento que existe actualmente acerca de estas especies y su hábitat se concentra en $P$. sericea en ecofisiología (Rada et al. 1985; Squeo et al. 1991; Azócar and Rada 1993; Rada et al. 2011), biogeografía (Kerr 2004; Zutta et al. 2012), diversidad faunística asociada (Valderrama and Verhelst 2009) y estructura de la vegetación (Hueck 1960; León 1991; Rangel-Ch and ArellanoP. 2010). Por su parte, el conocimiento de $P$. incana se centra en biogeografía, manejo, genética y autoecología (Vásquez et al. 2014; Duchicela et al. 2006; Catalano et al. 1995). En cambio, la información sobre $P$. quadrijuga es más escasa, con pocos trabajos puntuales en avifauna asociada (Meneses and HerreraMartínez 2013), ecofisiología (Ramos et al. 2013) y rasgos funcionales (Vélez et al. 1998). La distribución geográfica de estas especies en territorio colombiano se abordó sólo para $P$. sericea en páramos de la cordillera Central y para $P$. quadrijuga en la cordillera Oriental y en el páramo de Frontino en la cordillera Occidental (Fandiño and Caro-Sabogal 2009), pero no se hace mención alguna sobre la presencia de $P$. incana en el país (Fandiño and Caro-Sabogal 2009; Valderrama and Verhelst 2009; Rangel-Ch and Arellano-P. 2010). Además, el estado de conservación de las tres especies en Colombia nunca fue evaluado (Pérez 2017).

Dado que el monitoreo de las especies raras o amenazadas es una prioridad para diferentes países (López-Gallego 2015) y organizaciones conservacionistas (Guisan et al. 2006), la modelización del nicho ecológico de las especies y de su distribución geográfica puede contribuir a enfocar los esfuerzos, pues esta es una herramienta que permite proyectar el área potencial que ocupa una especie (Guisan et al. 2006; Carnaval and Moritz 2008) e identificar sitios y requerimientos ambientales adecuados para la supervivencia de las poblaciones (Soberón and Nakamura 2009; Soberón 2010). Esta información es básica para evaluar el estado de conservación de las especies (Thorn et al. 2009), para planificar estrategias de conservación y para seleccionar áreas de protección (Pearce and Boyce 2006; Papeş and Gaubert 2007). Como ejemplo, alrededor del mundo se desarrollaron diferentes investigaciones en modelización de la distribución de especies amenazadas de flora (Kumar and Stohlgren 2009; LópezGallego 2015) y fauna (Hernández et al. 2008; $\mathrm{Hu}$ and Jiang 2010; Toranza et al. 2016). Asimismo, la información de estos modelos puede ser útil para plantear la re-introducción de poblaciones en proyectos de restauración ecológica, o para manejar mejor las colecciones vivas de estas especies, entre otras aplicaciones (Elith et al. 2006). De acuerdo con lo anterior, en este trabajo se plantearon como objetivos identificar y analizar la distribución geográfica potencial de $P$. incana, $P$. quadrijuga y P. sericea, así como describir cuantitativamente sus oportunidades de conservación en el territorio colombiano.

\section{Materiales y Métodos}

\section{Área de estudio}

Para la recopilación de registros geográficos se contemplaron todas las aquellas zonas montañosas de los Andes de América del Sur que forman parte de la distribución de presencia de $P$. incana, $P$. quadrijuga y $P$. sericea. No obstante, los resultados de la modelización se presentan sólo para las zonas de alta montaña de los Andes colombianos por encima de $2800 \mathrm{~m} \mathrm{~s}$. n. m., que se dividen en Cordillera Oriental, Cordillera Central y Cordillera Occidental. El sistema montañoso conocido como Serranía de Perijá se incluyó como parte de la Cordillera Oriental. Además, se incluyó la Sierra Nevada de Santa Marta, aunque allí no se registró ninguna de las tres especies de Polylepis hasta el momento. Si bien las variables climáticas utilizadas se encuentran disponibles para toda la superficie 
terrestre en la página de Internet WorldClim 1.4 (www.worldclim.org), se seleccionó un área de estudio pertinente según la distribución real actual de las tres especies de Polylepis estudiadas, que comprende, en sentido amplio, el área continental de Sudamérica desde $23^{\circ} \mathrm{S}$ hasta $13^{\circ} \mathrm{N}$, e incluye desde Colombia y Venezuela hasta Bolivia en el extremo meridional. P. quadrijuga es una especie endémica de Colombia (Pérez 2017), P. incana se encuentra sólo en Ecuador y en Colombia, y $P$. sericea se registró desde Venezuela hasta el norte y centro de Bolivia (Zutta et al. 2012).

\section{Obtención de datos}

Los dos tipos de variables usadas en la modelización del nicho ecológico fueron los registros geográficos de presencia de las especies y las capas geográficas de las variables climáticas y geofísicas disponibles para el área de estudio.

Registros biológicos. Se descargaron y se depuraron 223 registros biológicos contenidos en bases de datos especializadas en biodiversidad (GBIF, SIB Colombia y Herbario COL). En el caso de P. sericea, abarcan localidades de Bolivia, Perú, Ecuador, Colombia y Venezuela. Se obtuvieron 96 reportes georreferenciados de $P$. incana, 32 de P. quadrijuga y 95 de $P$. sericea. La organización y la depuración de los datos se realizó con el programa ModestR Data Manager Version 3.2 (García-Roselló et al. 2014).

Variables ambientales. Se utilizaron 19 variables climáticas obtenidas del portal WorldClim 1.4 (www.worldclim.org), denominadas BIO1 a BIO19 (Tabla 1), descargadas para el tiempo actual con una resolución de 30 arco-segundos por pixel. Esta información se obtiene a partir de la interpolación de datos climáticos de 50 años (1960-1990) para todo el planeta (Hijmans et al. 2005). Además, se calcularon cuatro variables topográficas (altitud, pendiente, aspecto y curvatura) a partir del modelo digital de elevación "SRTM 90m Digital Elevation Database v4.1", producido originalmente por la NASA (Jarvis et al. 2008), para un total de 23 variables geográficas ambientales. Finalmente, se generaron cortes de las 23 variables ambientales en formato raster ASCII con una resolución de $0.504^{\prime} \times 0.504^{\prime}$ geográficos por pixel $\left(\sim 0.85 \mathrm{~km}^{2} /\right.$ pixel$)$.

Tabla 1. Las 23 variables ambientales seleccionadas y su porcentaje de contribución en la modelización en MaxEnt de la distribución geográfica potencial de las especies colombianas del género Polylepis. Las unidades de cada variable se pueden consultar en el portal web de WorldClim (Hijmans et al. 2005). Tres variables no fueron evaluadas (NE) para $P$. quadrijuga por su alta correlación en el modelo.

Table 1. The 23 selected environmental variables and their percent contribution in MaxEnt model the potential geographic distribution of the Colombian species of the genus Polylepis. The units of each variable can be consulted on the WorldClim web portal (Hijmans et al. 2005). Three variables were not evaluated (NE) for P. quadrijuga because of their high correlation in the model.

\begin{tabular}{|c|c|c|c|c|}
\hline Variable & Descripción & $\begin{array}{c}\text { Contribución } \\
\text { de } P \text {. incana al } \\
\text { modelo (\%) }\end{array}$ & $\begin{array}{l}\text { Contribución de } \\
\text { P. quadrijuga al } \\
\text { modelo (\%) }\end{array}$ & $\begin{array}{c}\text { Contribución } \\
\text { de } P \text {. sericea al } \\
\text { modelo }(\%)\end{array}$ \\
\hline BIO1 & Temperatura media anual & 0.8 & 6 & 0 \\
\hline $\mathrm{BIO} 2$ & Rango Medio (media mensual [max temp-min temp]) & 0.4 & 12.4 & 0.9 \\
\hline $\mathrm{BIO} 3$ & Isotermalidad (BIO2/BIO7) $\left({ }^{*} 100\right)$ & 10.7 & 4.6 & 0 \\
\hline $\mathrm{BIO} 4$ & Temperatura estacional (desviación estándar *100) & 12.6 & 17.4 & 22.2 \\
\hline BIO5 & Temperatura máxima del mes más caliente & 0.9 & 33.6 & 0.3 \\
\hline BIO6 & Temperatura mínima del mes más frío & 1.5 & 2.6 & 0.4 \\
\hline $\mathrm{BIO7}$ & Rango de temperatura anual (BIO5-BIO6) & 12.5 & 0.2 & 0 \\
\hline BIO8 & Media de la temperatura del trimestre más húmedo & 0 & 0.2 & 0.1 \\
\hline BIO9 & Media de la temperatura del trimestre más seco & 0 & 0.1 & 0 \\
\hline BIO10 & Media de la temperatura del trimestre más caliente & 0 & 14.8 & 0 \\
\hline BIO11 & Media de la temperatura del trimestre más frío & 0 & 0.4 & 0.7 \\
\hline BIO12 & Precipitación anual & 0 & 0.1 & 0 \\
\hline $\mathrm{BIO13}$ & Precipitación del mes más húmedo & 0 & NE & 0 \\
\hline BIO14 & Precipitación del mes más seco & 0 & 0.1 & 0.1 \\
\hline BIO15 & Precipitación estacional (Coeficiente de variación) & 0 & 0 & 0 \\
\hline BIO16 & Precipitación del trimestre más húmedo & 0 & NE & 0 \\
\hline BIO17 & Precipitación del trimestre más seco & 1 & 2.5 & 0.8 \\
\hline BIO18 & Precipitación del trimestre más caliente & 0 & 0.1 & 0.1 \\
\hline BIO19 & Precipitación del trimestre más frío & 0.4 & 0.7 & 0.5 \\
\hline Altitud & Altura sobre el nivel del mar & 58.9 & NE & 64.9 \\
\hline Pendiente & Grados de pendiente del terreno & 0.1 & 3.2 & 6.7 \\
\hline Aspecto & Dirección principal de la pendiente del terreno & 0.2 & 0.5 & 0.1 \\
\hline Curvatura & Derivada de la pendiente, terreno cóncavo o convexo & 0.1 & 0.4 & 2 \\
\hline
\end{tabular}


Se usó el factor de inflación de la varianza (VIF, por sus siglas en inglés) para seleccionar las variables no correlacionadas a partir de una matriz con las 23 variables, donde los valores de VIF mayores a 10 indican problemas de multicolinealidad de esa variable en cada modelo con respecto al resto de las variables evaluadas (Myers 1990). De esta manera se decidió incluir las 23 variables para los modelos de $P$. incana y $P$. sericea, mientras que para $P$. quadrijuga descartaron las variables de BIO13, BIO16 y altitud (Tabla 1).

\section{Análisis de la información geográfica}

Modelización de la distribución potencial. Para estimar la distribución geográfica potencial se realizó la modelización del nicho ecológico de las tres especies en el programa MaxEnt (Maximum Entropy Species Distribution Modeling) versión 3.3.3k (Elith et al. 2011). MaxEnt modela la distribución geográfica potencial de las especies a partir de la ubicación geográfica de presencias conocidas y las variables expresadas en capas de información ambiental (que pueden ser continuas o categóricas) asociadas a estos puntos. Esto le permite hacer transiciones entre el espacio geográfico y el espacio ecológico para modelizar la distribución de las especies (Elith et al. 2011; Phillips et al. 2006).

Para las tres especies, se empleó el método de Bootstrap con 100 repeticiones en MaxEnt y se tomó una submuestra aleatoria de $20 \%$ de los puntos para probar el modelo en cada repetición. Al final, se usaron los resultados promedio de las repeticiones, expresados espacialmente en una escala logística de probabilidad. Para considerar una alta probabilidad de presencia de las condiciones ambientales que describen el nicho de la especie, se utilizó un valor de corte independiente para cada especie modelada, tomando siempre el valor del umbral logístico de la prueba equilibrada de sensibilidad y especificidad (equal test of sensitivity and specificity logistic threshold), reportado por el programa MaxEnt para cada especie (Padonou et al. 2015; Liu et al. 2005).

Identificación de oportunidades de conservación. Para analizar las oportunidades de conservación de las especies de Polylepis presentes en Colombia, se partió de los mapas de distribución potencial obtenidos en MaxEnt, el mapa de delimitación de complejos de páramo a escala 1:100.000 para Colombia (Sarmiento-Pinzón et al. 2013) y las áreas protegidas incluidas en la página del SIAC (www.ideam.gov.co/web/siac). Con el fin de calcular las áreas de superposición entre un área protegida y la distribución potencial ajustada de cada especie se incluyeron las siguientes categorías de áreas protegidas: Parque Nacional Natural, Parque Natural Regional, Distrito de Conservación de Suelos, Distrito Regional de Manejo Integrado, y Reserva Natural de la Sociedad Civil.

\section{Resultados}

\section{Modelización de la distribución geográfica}

Las variables que más contribuyen en general a los tres modelos de distribución son altitud, temperatura estacional (BIO4), rango de temperatura media mensual (BIO2) y rango anual de temperatura (BIO7). Sin embargo, el porcentaje de contribución de cada una de las variables ambientales es diferente para cada especie (Tabla 1).

Para P. incana, se utilizó el umbral de 0.701 de probabilidad de ocurrencia al establecer su área de distribución potencial. El resultado son $1772.8 \mathrm{~km}^{2}$ en la Cordillera Central y la frontera sur del país, que corresponde a su área de distribución real verificada entre Colombia y Ecuador, en el páramo del volcán de Chiles y en el área de influencia del volcán Cumbal, en el departamento de Nariño, además de una zona en la Cordillera Central donde no hay reportes de la especie (Figura 1). Los máximos valores de probabilidad de ocurrencia de $P$. incana se dan entre 3400 y $4000 \mathrm{~m}$ s. n. m. Al observar por separado las variables de temperatura estacional (BIO4) y de rango anual de temperatura (BIO7), que son las que más contribuyen al modelo (Tabla 1), se encontró que la especie prefiere una variación muy pequeña de temperaturas, con una probabilidad máxima de encontrarla en áreas sin estaciones $(\mathrm{BIO} 4=0)$ y con una diferencia de $10^{\circ} \mathrm{C}$ entre la máxima temperatura del año y la mínima $(B I O 7=100)$. Esto indica que P. incana se puede presentar con mayor probabilidad en zonas con muy poca diferencia de temperatura entre las estaciones.

Para establecer el área de distribución potencial de $P$. quadrijuga se utilizó el umbral de corte 0.5546 de probabilidad de ocurrencia. $P$. quadrijuga presentó un área potencial de $6264.2 \mathrm{~km}^{2}$ ubicados en la Cordillera Oriental en los departamentos de Santander y Norte de Santander en el complejo de páramos 


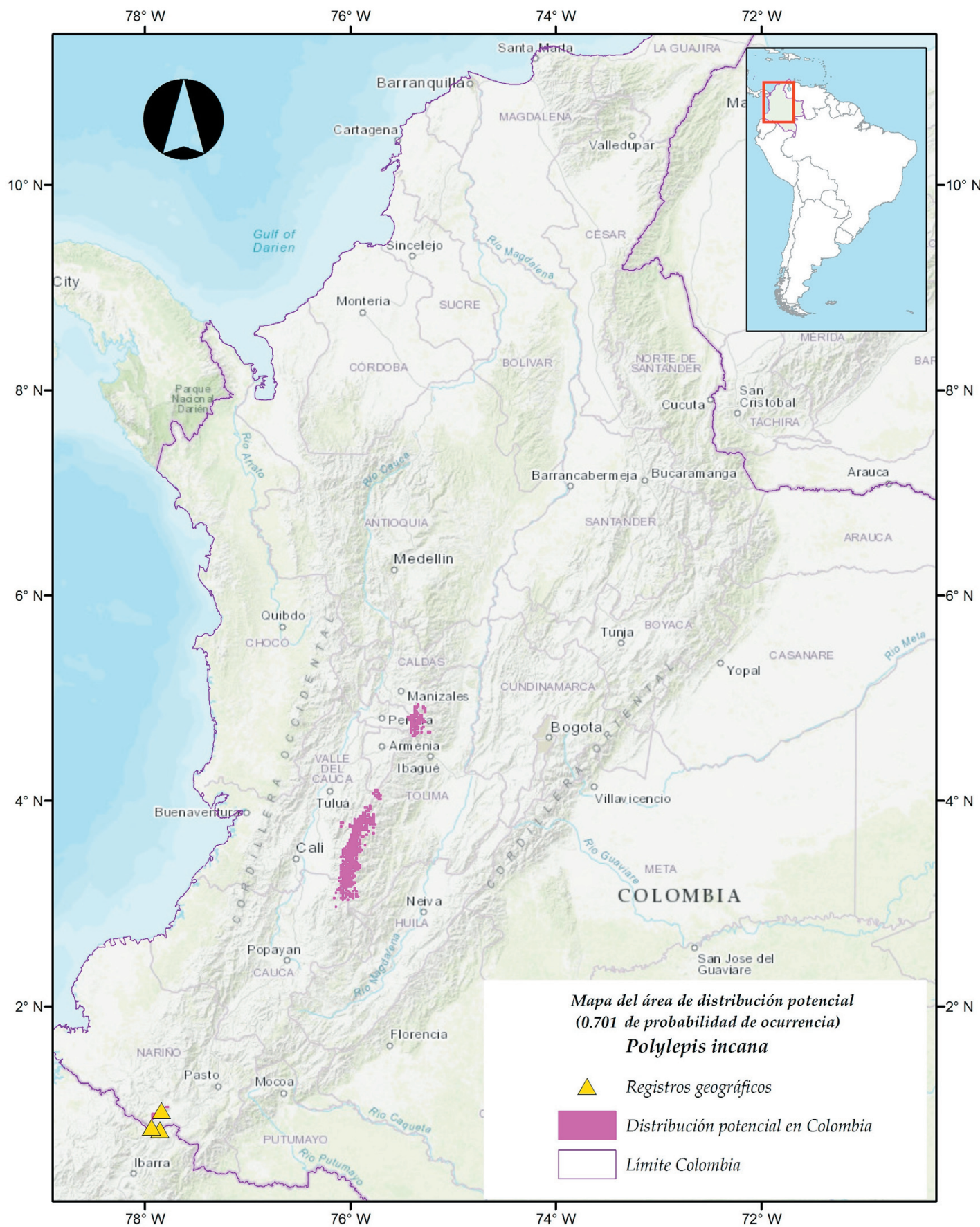

Figura 1. Mapa de distribución potencial (0.701 de probabilidad de ocurrencia) para Polylepis incana en Colombia. Figure 1. Map of potential distribution (0.701 probability of occurrence) for Polylepis incana in Colombia.

de Berlín, Santurbán y Almorzadero, en el departamento de Boyacá en el Parque Nacional Natural (PNN) El Cocuy y otros complejos de páramos, en Cundinamarca en el PNN Chingaza y otros complejos de páramos $\mathrm{y}$ tangencialmente algunas zonas de Meta y Huila como parte del páramo de Sumapaz
(Figura 2). Las variables que más influyen sobre el modelo de distribución potencial de $P$. quadrijuga son la temperatura máxima del mes más caliente (BIO5) (soportando un valor máximo de $12{ }^{\circ} \mathrm{C}$ ), la temperatura estacional con mayores valores de probabilidad a cero grados (una baja desviación estándar de la 


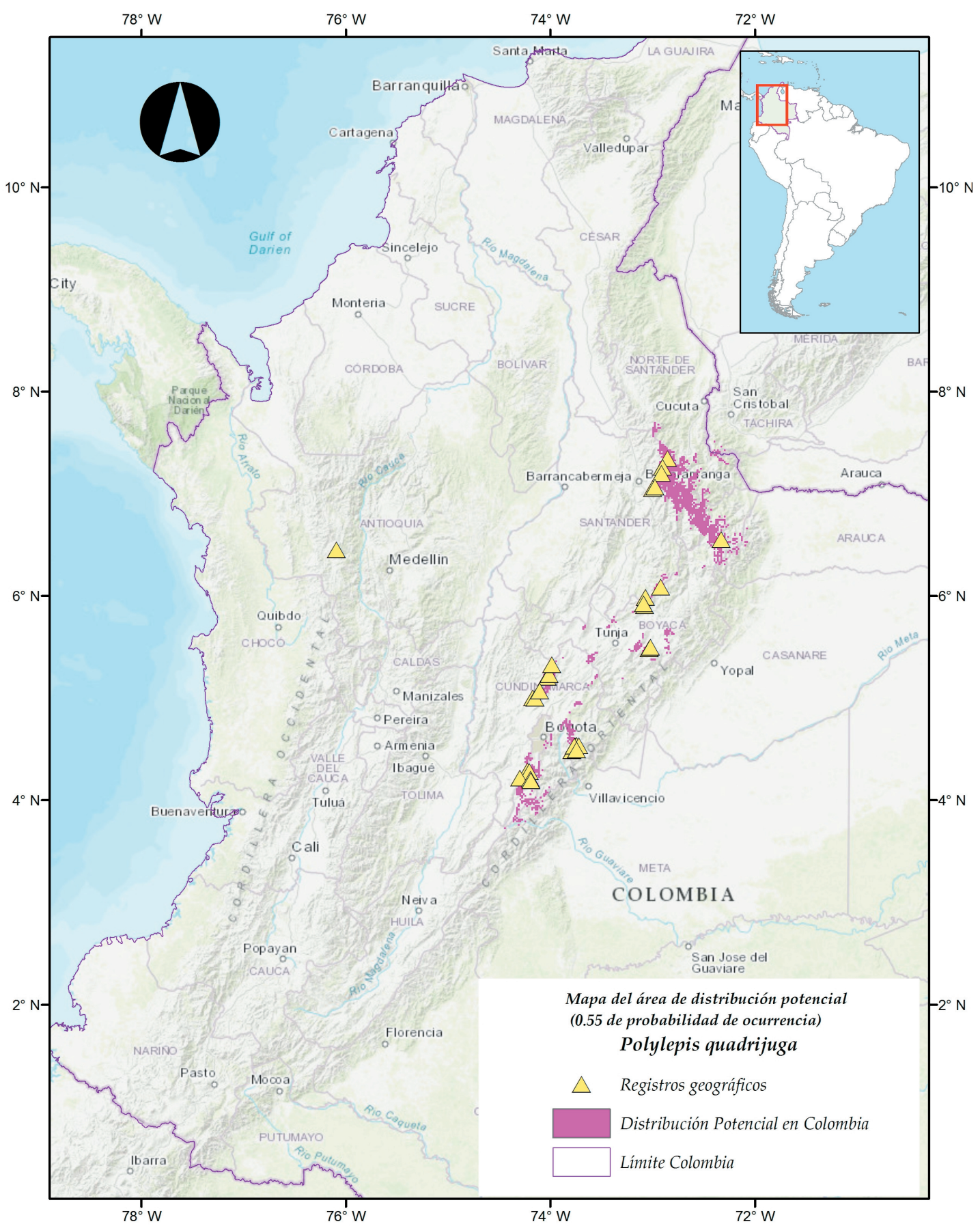

Figura 2. Mapa de distribución potencial (0.55 de probabilidad de ocurrencia) para Polylepis quadrijuga en Colombia. Figure 2. Map of potential distribution (0.55 probability of occurrence) for Polylepis quadrijuga in Colombia.

temperatura $\mathrm{BIO} 4=0$ ), $\mathrm{BIO} 10$, con valores de la temperatura media del trimestre más cálido cercanos a $8^{\circ} \mathrm{C}$ y Rango de temperatura media mensual (BIO2), con altos valores de probabilidad $(\mathrm{BIO} 2=80)$ de $8^{\circ} \mathrm{C}$ de diferencia entre la temperatura media del mes más cálido y la media del mes más frío (Tabla 1).
Al establecer el área de distribución potencial para $P$. sericea, se utilizó el umbral de corte 0.53 de probabilidad de ocurrencia. Presentó el área de distribución potencial más amplia, con $23243.5 \mathrm{~km}^{2}$ en las tres cordilleras de Colombia y la Sierra Nevada de Santa Marta, con las áreas más extensas en los páramos de 


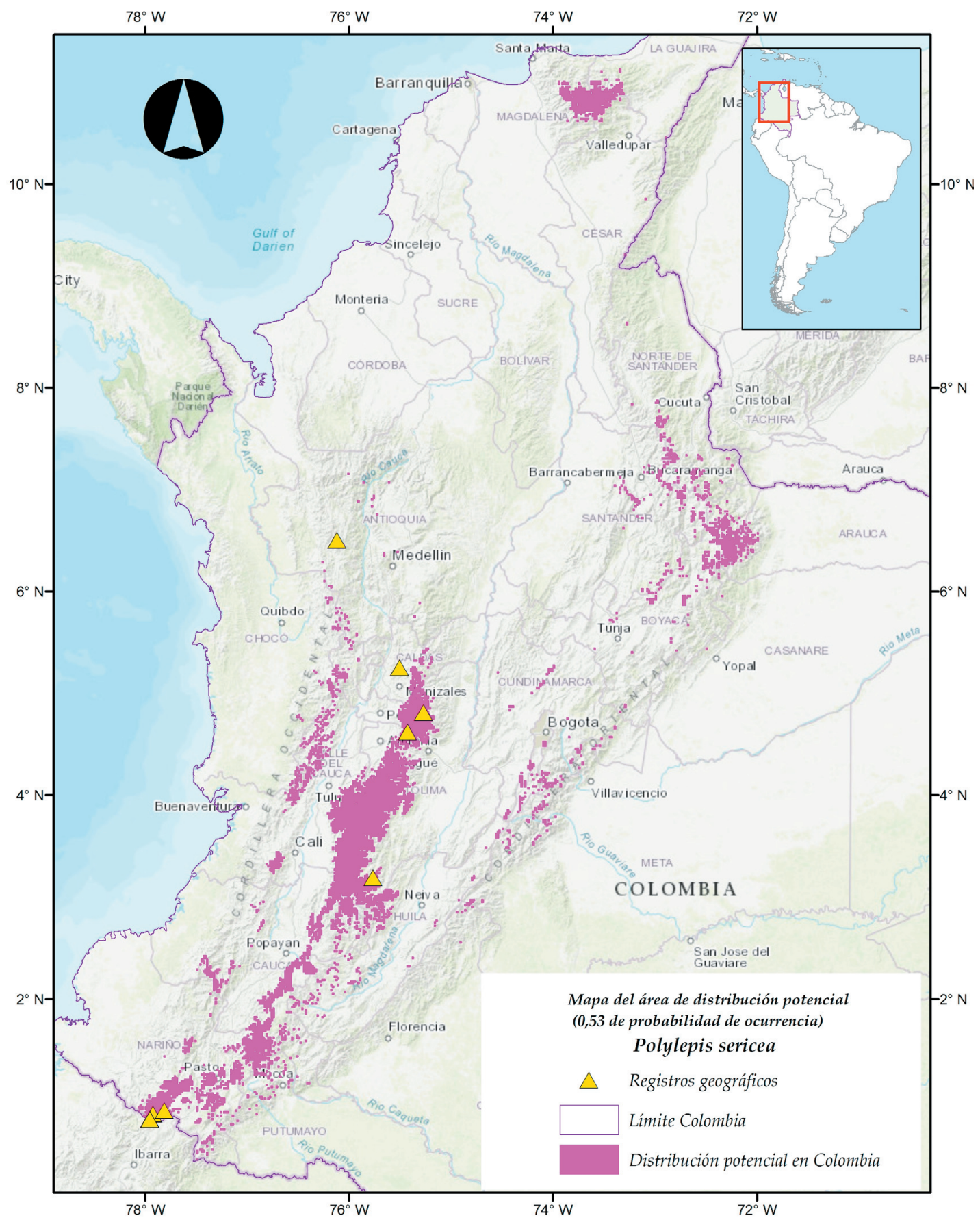

Figura 3. Mapa de distribución potencial (0.53 de probabilidad de ocurrencia) para Polylepis sericea en Colombia. Figure 3. Map of potential distribution (0.53 probability of occurrence) for Polylepis sericea in Colombia.

la cordillera Central en los departamentos de Nariño, Cauca, Valle del Cauca, Tolima, Quindío, Caldas y Risaralda (Figura 3). Las variables que más influyen sobre el modelo de distribución potencial de $P$. sericea son la altitud (con un pico hacia los 3300 m s. n. m. y manteniendo probabilidades altas hasta
4300 m s. n. m.) y BIO4 (con las mayores probabilidades en valores de 0 a $11^{\circ} \mathrm{C}$ de desviación estándar, lo cual implica una afinidad mayor por los climas con estaciones, al menos en parte de su distribución). La pendiente, que fue la tercera variable en importancia para este modelo (Tabla 1), 


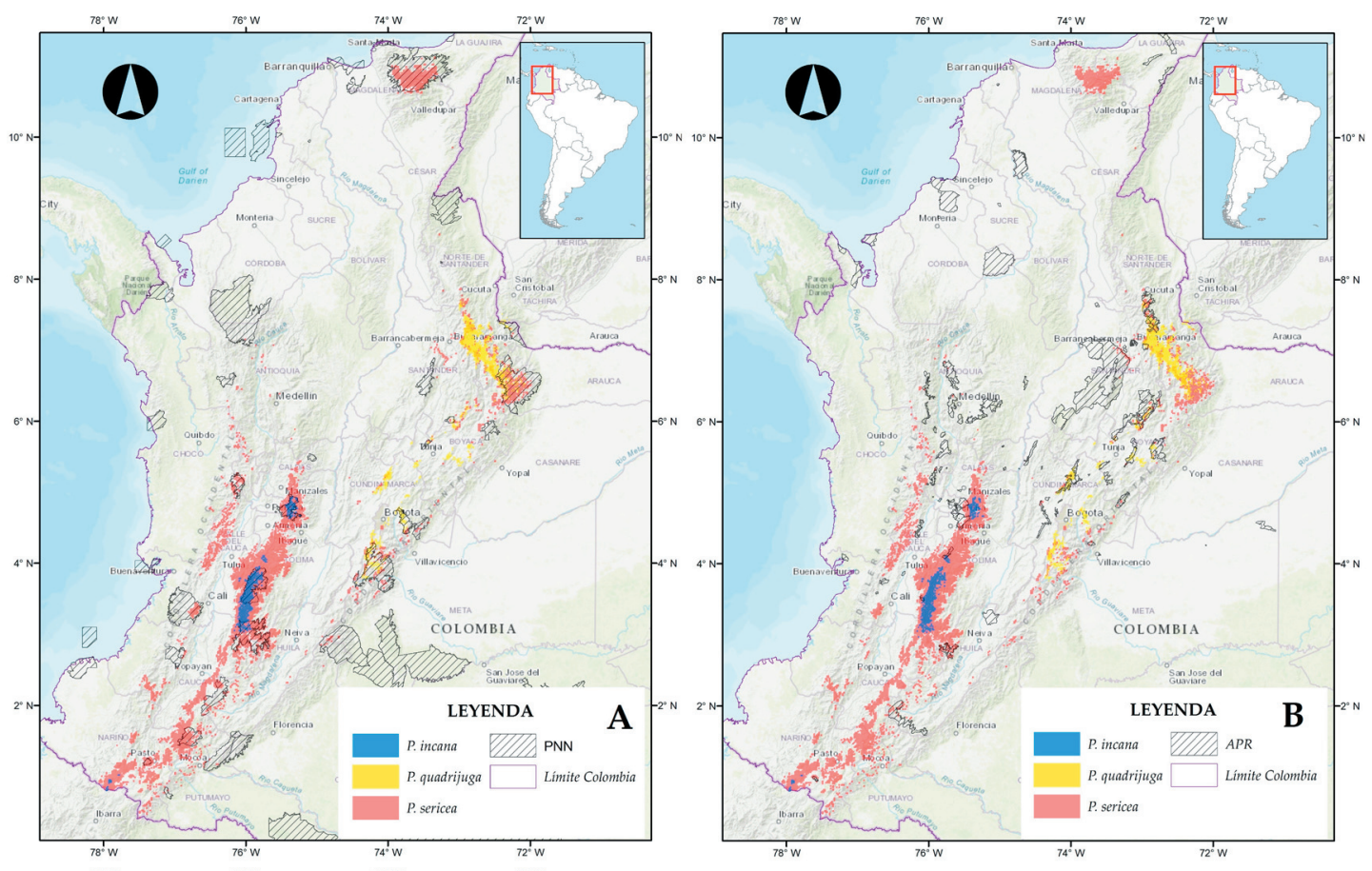

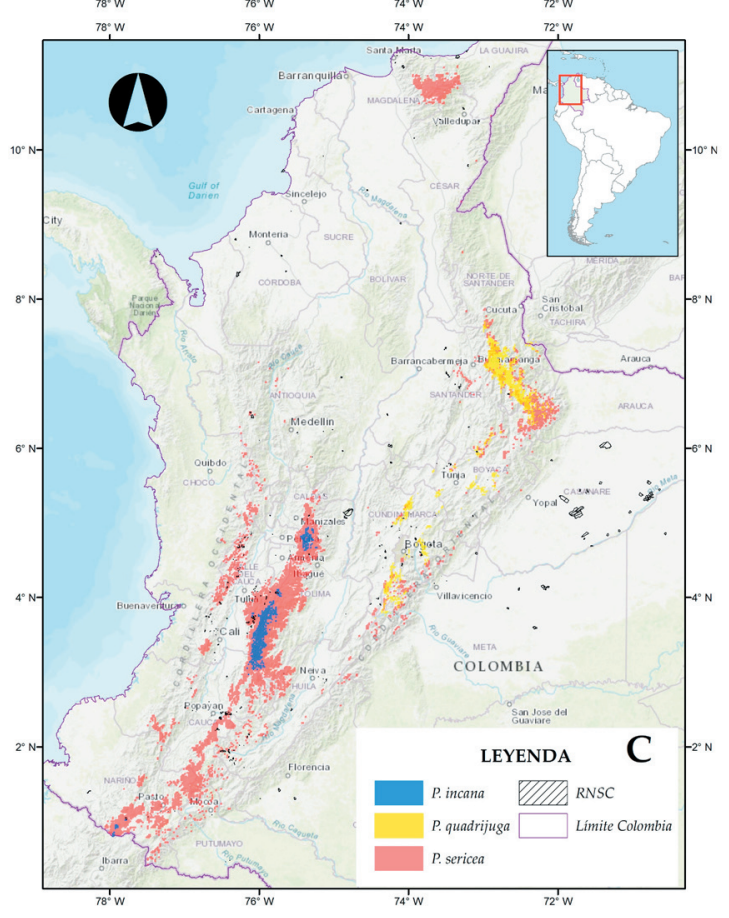

muestra sus mayores valores de probabilidad de ocurrencia de $P$. sericea en zonas inclinadas entre 10 y 30 grados.

\section{Oportunidades de conservación}

La mayor parte de la distribución potencial de las tres especies se superpone con áreas de conservación de carácter privado, regional
Figura 4. Análisis de coincidencia entre el área de distribución potencial de las tres especies de Polylepis y las áreas protegidas del SINAP en Colombia. A: parques nacionales naturales (PNN); B: áreas protegidas regionales (APR); C: reservas naturales de la sociedad civil (RNSC).

Figure 4. Analysis of coincidence between the potential distribution area of the three Polylepis species and the protected areas of SINAP in Colombia. A: national natural parks (PNN); B: regional protected areas (APR); C: natural reserves of civil society (RNSC).

o nacional del Sistema Nacional de Áreas Protegidas (SINAP). P. incana tiene 59.5\% de su área de distribución potencial dentro de alguna figura de protección; no obstante, éstas no siempre son áreas protegidas del nivel nacional, sino locales o regionales (e.g., la Reserva Forestal Protectora (RFP) Volcán Azufral) (Figura 4B y 4C). En cambio, en los parques PNN Las Hermosas y PNN Los 
Tabla 2. Áreas y porcentajes de distribución geográfica potencial de las tres especies colombianas de Polylepis, incluidas en áreas protegidas y reservas naturales de los ámbitos nacional, regional y privado del SINAP.

Table 2. Areas and percentages of the potential geographic distribution of the three Colombian species of Polylepis, included in protected areas and natural reserves of the national, regional and private levels of SINAP.

\begin{tabular}{|c|c|c|c|c|c|c|c|}
\hline \multirow[b]{2}{*}{ Ámbito } & \multirow[b]{2}{*}{ Figura de protección } & \multicolumn{2}{|c|}{$\begin{array}{l}\text { P. incana } \\
\text { ADP: } 1772.8 \mathrm{~km}^{2}\end{array}$} & \multicolumn{2}{|c|}{$\begin{array}{l}\text { P. quadrijuga } \\
\text { ADP: } 6264.2 \mathrm{~km}^{2}\end{array}$} & \multicolumn{2}{|c|}{$\begin{array}{l}\text { P. sericea } \\
\text { ADP: } 23243.5 \mathrm{~km}^{2}\end{array}$} \\
\hline & & $\begin{array}{l}\text { Área } \\
\text { protegida } \\
\left(\mathrm{km}^{2}\right)\end{array}$ & $\%$ & $\begin{array}{l}\text { Área } \\
\text { protegida } \\
\left(\mathrm{km}^{2}\right)\end{array}$ & $\%$ & $\begin{array}{l}\text { Área } \\
\text { protegida } \\
\left(\mathrm{km}^{2}\right)\end{array}$ & $\%$ \\
\hline Nacional & Parque Nacional Natural & 1027.07 & 57.9 & 1818.23 & 29.0 & 6797.79 & 29.2 \\
\hline Regional & Parque Natural Regional & 11.04 & 0.6 & 686.01 & 11.0 & 570.98 & 2.5 \\
\hline Regional & $\begin{array}{l}\text { Distrito de Conservación de } \\
\text { Suelos }\end{array}$ & 0.21 & 0.01 & 0.0 & 0.0 & 44.39 & 0.2 \\
\hline Regional & $\begin{array}{l}\text { Distrito Regional de Manejo } \\
\text { Integrado }\end{array}$ & 11.3 & 0.64 & 295.85 & 4.7 & 348.95 & 1.5 \\
\hline \multirow[t]{2}{*}{ Privado } & $\begin{array}{l}\text { Reserva Natural de la } \\
\text { Sociedad Civil }\end{array}$ & 4.7 & 0.27 & 6.23 & 0.1 & 65.47 & 0.3 \\
\hline & $\begin{array}{l}\text { ADP Total por especie en el } \\
\text { SINAP }\end{array}$ & 1054.8 & 59.5 & 2806.3 & 44.8 & 7833.0 & 33.7 \\
\hline
\end{tabular}

* ADP: Área de Distribución Potencial.

Nevados se encuentran áreas adecuadas para la especie, pero no se reportaron individuos de P. incana hasta el momento (Figura 4A).

P. quadrijuga muestra una menor cantidad de área potencial de distribución ubicada dentro de alguna figura de protección $(44.8 \%$ del área de distribución potencial). Estos sitios corresponden al complejo de páramo de Guerrero (IAvH 2012) en los municipios de Cogua, Tausa y Sutatausa, departamento de Cundinamarca, donde se tienen registros de la especie, en lo que se conoce como el Distrito de Manejo Integrado del Páramo de Guerrero, y al complejo de páramo del Almorzadero (IAvH 2012), entre los departamentos de Santander y Norte de Santander (Figura 4B).

Por su parte, el área de distribución potencial de $P$. sericea se encuentra protegida sólo en un $33.7 \%$ (Tabla 2) en el páramo de Frontino, cordillera Occidental, el PNN Los Nevados, y con un registro al norte del PNN Nevado del Huila. Según el modelo resultante es probable que se encuentre también, o que haya existido en algún momento, en el PNN Las Hermosas, PNN Puracé y PNN Doña Juana, que son áreas protegidas del nivel nacional, donde la especie no fue reportada aún. Las poblaciones presentes en el complejo de páramos ChilesCumbal (IAvH 2012) y las áreas probables de distribución cercanas en el departamento de Nariño no se encuentran protegidas, y concentran la mayor parte de los $15410 \mathrm{~km}^{2}$ (el restante $66.3 \%$ ) aproximadamente en donde la distribución potencial de $P$. sericea está por fuera del SINAP (Tabla 2).

\section{Discusión}

\section{Distribución geográfica potencial de Polylepis} en Colombia

La especie que se encuentra en la Cordillera Oriental es $P$. quadrijuga. Su origen evolutivo parece estar en la raíz de la filogenia del género, por lo que se ha propuesto a Colombia como el sitio de origen de Polylepis. En la propuesta de Kerr (2004), se señala el origen del género Polylepis en la hibridización alopoliploide entre Acaena elongata L. y Acaena cylindristachya Ruiz and Pav. Estas especies de Acaena aún convergen en la Cordillera Oriental colombiana y comparten su hábitat con $P$. quadrijuga, lo cual convierte a $P$. quadrijuga en una pieza clave en la historia natural del género.

El modelo resultante para $P$. quadrijuga muestra una distribución potencial muy cercana a la extensión de presencia observada, a pesar de que su área de ocupación es mucho menor en los departamentos de Santander y Norte de Santander, donde se predice una amplia zona de hábitat adecuado en los 
páramos de Santurbán y del flanco norte de la Sierra Nevada del Cocuy. En cambio, en los departamentos de Boyacá y Cundinamarca está restringida a los principales macizos de páramo (Figura 2). La presencia de poblaciones en el páramo de Frontino es remarcable, dado que la Cordillera Central y los valles de los ríos Cauca y Magdalena, por sus climas cálidos y ambientes tropicales secos o húmedos, son barreras geográficas para la expansión de las poblaciones de especies propias de páramo (Carrizosa and Hernández 1990). Por tal motivo, es posible pensar en dos explicaciones hipotéticas a esta distribución disyunta: la primera es que los procesos evolutivos de hibridización de $A$. elongata y A. cylindristachya se hayan dado de igual manera tanto en el páramo de Frontino como en la Cordillera Oriental; la segunda es que algún agente de dispersión haya llevado semillas o propágulos desde las poblaciones más extensas de la Cordillera Oriental hasta este páramo de Frontino, que es relativamente pequeño y aislado. Es probable que un estudio detallado de la genética de las poblaciones de esta especie permita entender mejor este patrón geográfico.

Cabe resaltar que la distribución potencial de $P$. quadrijuga no se superpone con la de $P$. incana y que sólo coincide en algunas zonas puntuales de la Cordillera Oriental con $P$. sericea (Figuras 1, 2 y 3), de la cual no se tienen registros puntuales en la Cordillera Oriental. Esto indica que el nicho de estas especies está separado por algunas de las variables climáticas, como la temperatura máxima del mes más caliente (BIO5). Esta variable resulta clave en el modelo de $P$. quadrijuga, $\mathrm{y}$, en general, la baja tolerancia de la especie ante cambios estacionales en la temperatura probablemente le permita a $P$. quadrijuga ocupar los espacios disponibles de la mayor parte de la cordillera Oriental.

Los datos palinológicos indican que las formaciones densas de $P$. quadrijuga en el páramo aparecieron hace 2.4 a 2.2 Ma. Esta fecha es reciente con respecto a la formación de la Cordillera Oriental y su último gran levantamiento hace 4.5 a $3.5 \mathrm{Ma}$, momento en el que ya existían ambientes de páramo en las tres cordilleras (Van der Hammen and Hooghiemstra 1997). Desde entonces, la distribución de Polylepis en Colombia varió en función de las fluctuaciones climáticas. Por ejemplo, en la Sabana de Bogotá se presentó un pico de abundancia relativa entre 150000 y 140000 años antes del presente, seguido de una desaparición posterior de los cerros Orientales de Bogotá, hace 30000 años, en coincidencia con el momento de drenaje del antiguo lago de Bogotá y los cambios ambientales que este evento produjo (Mommersteeg 1998; Torres 2006; Rangel and Arellano 2010). En la zona de la laguna de Fúquene (límites entre Cundinamarca y Boyacá) se registró un aumento en la abundancia de polen de Polylepis en un período entre 45000 y 35000 años antes del presente, y luego descendió hasta casi desaparecer en la actualidad (Mommersteeg 1998). Hoy en día sólo quedan los fragmentos que se observan en la zona del páramo de Guerrero, al norte de Bogotá (Figura 2).

El modelo resultante para $P$. sericea muestra varias zonas donde la distribución potencial de la especie es mucho más amplia que la extensión de presencia (Figura 3). Tal es el caso de la Cordillera Central y de los páramos de la Sierra Nevada de Santa Marta, donde hasta el momento no se registró ninguna especie de este género (Rangel et al. 1982; Cleef and Rangel-Ch. 1984; Rangel-Ch. and Jaramillo 1984). Sin embargo, este modelo es más aproximado al panorama colombiano que el modelo propuesto por Zutta et al. (2012), que muestra una amplia distribución potencial de $P$. sericea en la Cordillera Oriental colombiana, lugar en donde no hay registros geográficos de esta especie (Pérez 2017). No obstante, cabe señalar que el área de distribución potencial resulta ser mucho mayor en el presente estudio $\left(22477 \mathrm{~km}^{2}\right)$ que en el área modelizada anteriormente, que abarcaba $10324 \mathrm{~km}^{2}$ en Colombia (Zutta et al. 2012). En este caso, la ausencia de registros de $P$. sericea en la Cordillera Oriental de Colombia permite establecer una mejor aproximación a la distribución real en este estudio que en el de Zutta et al. (2012). El área de distribución potencial que se encuentra en el sector norte de la Sierra Nevada del Cocuy y en los complejos de páramos de Berlín y Almorzadero (Figura 3) es bastante más amplia de lo que puede ser su distribución actual. De esta manera, queda abierta la incógnita de si esta especie se puede presentar realmente en esta zona, pues, aunque no hay registros biológicos confirmados para la Cordillera Oriental colombiana, $P$. sericea es la única que se encuentra en la Cordillera de Mérida en Venezuela, con un origen geológico similar y una continuidad geográfica evidente. Esta distribución disyunta de $P$. sericea, que contiene o encierra a la distribución de la especie basal del grupo ( $P$. quadrijuga), y la amplia distribución de $P$. sericea en los países andinos hasta Bolivia, lleva a pensar en el 
clado infragenérico Sericea como el grupo descendiente más cercano a $P$. quadrijuga, como un siguiente paso en la compleja historia natural del género cuyas adaptaciones le permitieron desplazarse hacia el sur por los Andes (Segovia 2014).

Para $P$. incana se excluyen de la distribución potencial los alrededores del Nevado del Ruiz, en el PNN Los Nevados (Figura 1), dado que las colecciones botánicas y los estudios bióticos con los que se cuenta para la zona no reportaron la presencia de esta especie hasta el momento (Cleef et al. 1983; Rangel-Ch. et al. 1983). Además, el modelo señala áreas de distribución potencial muy poco exploradas en el complejo de páramos entre Tolima y Valle del Cauca, con su límite sur en el Nevado del Huila (Figura 1). Esto abre las posibilidades a una exploración botánica de esta zona de páramo que, en general, posee pocos registros. Por otra parte, se evidencia que el área de distribución potencial de $P$. incana en Colombia se incluye dentro del área de $P$. sericea, que se consideran especies hermanas en algunos análisis filogenéticos (Segovia 2014), tanto en Nariño como en la Cordillera Central, donde están muy superpuestas (Figuras 1 y 3).

Se propone realizar el estudio de la distribución geográfica de todas las especies del género Polylepis en colaboración con científicos de otros países, utilizando herramientas como la modelización del nicho ecológico y otras técnicas comparables. Una hipótesis de trabajo dentro de este marco de investigación es que las especies que colonizaron el sur del continente, partiendo desde la alta montaña intertropical, lograron adaptarse en menor o mayor medida a los cambios estacionales en la temperatura y a diferentes condiciones ambientales de humedad y altitud, que no existían en sus rangos geográficos ancestrales.

\section{Aspectos de conservación en Colombia}

La presión antrópica sobre los bosques de Polylepis es similar a lo largo de los Andes colombianos, supeditada a las presiones típicas del páramo (e.g., la extracción de leña, la ganadería extensiva con las quemas asociadas y la transformación de coberturas naturales en cultivos de papa [Rangel-Ch. and Arellano-P. 2010]). La presencia de este tipo de disturbios hace de los páramos un paisaje cada vez más homogéneo, en donde empiezan a predominar los pastos con crecimiento en macolla y las rosetas, que resisten al fuego (Verweij 1995; Vargas 2002; Vargas 2013). Sin embargo, ni la vegetación general ni el suelo están adaptados a resistir fuego y pastoreo de forma permanente (Vargas 2013). Para el caso de las coberturas de bosques dominadas por P. quadrijuga, Rangel-Ch. y Arellano (2010) encontraron que aquellas zonas que alguna vez estuvieron arboladas se transformaron en áreas agropecuarias donde la recuperación de la vegetación puede ser muy difícil en el largo plazo. Esto hace que la reducción de los bosques de Polylepis se convierta en una pérdida irreparable, pues las poblaciones de esta especie se han reducido drásticamente desde el último período glacial. Aunque las razones de esta reducción aún no son claras, lo cierto es que las acciones antropogénicas la acentuaron (Van der Hammen 1986). Esto mismo también se podría aplicar a $P$. sericea y a $P$. incana, ya que la ocupación humana de las tres cordilleras colombianas ha ejercido una presión creciente desde tiempos precolombinos y hoy en día es todavía más grave por las nuevas prácticas agrícolas y por el acceso a lugares antiguamente muy remotos de la geografía colombiana.

Aunque la distribución potencial de $P$. incana presenta el mayor porcentaje de áreas de conservación (Tabla 2), su distribución potencial y los registros de la especie se encuentran en una zona muy pequeña y de importancia para las actividades productivas, principalmente agropecuarias. Por su parte, $P$. quadrijuga es la que corre el mayor riesgo dado que cuenta con un porcentaje menor de su distribución dentro de áreas protegidas y es endémica de la Cordillera Oriental. En cambio, $P$. sericea cuenta con más oportunidades de conservación al tener una mayor área de su distribución potencial en el país dentro del sistema de áreas protegidas (Tabla 2), sumado a que es una distribución amplia dentro y fuera del territorio nacional de Colombia. Teniendo esto en cuenta, $P$. quadrijuga es la especie que requiere una atención especial en términos de conservación. Tomando en cuenta los resultados de este trabajo, se recomienda enfocar los esfuerzos de conservación y de manejo en las poblaciones del Páramo de Guerrero y del complejo de páramos Santurbán-Berlín-Almorzadero, en los departamentos de Santander y Norte de Santander, en donde no hay áreas protegidas del SINAP (Figura 4).

La verificación de campo también es necesaria en algunos casos como las poblaciones del PNN Sumapaz, pues las áreas potenciales de bosques de Polylepis 
presentadas por Fandiño and Caro-Sabogal (2009) en esta zona no coinciden con las áreas que los autores han encontrado en algunos sectores del páramo de Sumapaz (observación personal), como las áreas cercanas al centro poblado del corregimiento de San Juan del Sumapaz y la cuenca alta del río Santa Rosa, en el corregimiento de Nazareth. Dado que no existe una categorización de amenaza de ninguna de las tres especies en el país (Pérez 2017), no es posible asegurar que $P$. incana y $P$. sericea se encuentren en un buen estado de conservación. La única aproximación es la propuesta de categorización de amenaza de los tipos de vegetación dominados por Polylepis de Rangel-Ch and Arellano-P. (2010), que se basa sobre la extensión de los parches y la fragmentación por urbanismo o por uso agrícola del suelo, aunque en aquel entonces se desconocía la presencia de $P$. incana en el sur del país y no se hace ninguna referencia a los bosques de esta especie. Por ello, se propone usar los registros geográficos recopilados y los modelos elaborados en la presente contribución como insumo para realizar la categorización de amenaza de estas especies según criterios de reducción de su distribución geográfica que utiliza la IUCN (2017), es decir, extensión de presencia y área de ocupación.

Dos campos de investigación que también se deben abordar, de manera paralela a la categorización del grado de amenaza, son los estudios de la genética poblacional de las tres especies y sus flujos génicos, así como la proyección del cambio de las coberturas de bosques de Polylepis frente a escenarios inminentes de cambio climático. A pesar de esto, todas estas herramientas académicas deben tener un respaldo en las decisiones gubernamentales e institucionales, de forma tal que se puedan emprender acciones de conservación y de restauración para impedir la desaparición de los bosques de Polylepis, en especial de $P$. quadrijuga, la única especie del género que es endémica de Colombia.

La especie que presenta el mejor panorama de conservación es $P$. incana, dado que su distribución en el país ocupa un área reducida, aunque protegida en mayor medida por el SINAP, mientras que $P$. quadrijuga es la que se encuentra más afectada debido a una distribución potencial poco representada dentro de áreas protegidas y con la presencia de múltiples amenazas en una gran parte de su extensión. Los modelos de distribución obtenidos aportan significativamente al conocimiento de los Polylepis y se recomienda tenerlos en cuenta en futuras actividades de investigación y manejo para su conservación y restauración.

Agradecimientos. Al equipo de Sistemas de Información Geográfica de la Subdirección Científica del Jardín Botánico de Bogotá José Celestino Mutis, en especial a Claudia Huertas García, Liliana Corzo Ramírez y Oscar Rojas Zamora; al comité organizador del IV Congreso Internacional de Ecología y Conservación de Bosques de Polylepis, especialmente a Erica Cuyckens, Gustavo Guzmán y Daniel Renison; y a Yoluka ONG Fundación de investigación en biodiversidad y conservación.

\section{REFERENCIAS}

Azócar, A., and F. Rada. 1993. Ecofisiología de plantas de alta montaña andina. Pp. 82-110 in A. Azócar (ed.) Respuestas ecofisiológicas de plantas de ecosistemas tropicales. Ediciones del CIELAT, Universidad de los Andes, Mérida, Venezuela.

Carnaval, A. C., and C. Moritz. 2008. Historical climate modelling predicts patterns of current biodiversity in the Brazilian Atlantic forest. J Biogeogr 35:1187-1201.

Carrizosa, J., and C. Hernández (eds.). 1990. Selva y Futuro: Colombia. Bogotá: El Sello Editorial. Pp. 151-184.

Catalano, S. P., L. Cioni, M. Martinozzi, V. De Feo, and I. Morelli. 1995. Chemical investigation of Polylepis incana (Rosaceae). Biochem Syst Ecol 23(1):105-107.

Cleef, A. M., and J. O. Rangel-Ch. 1984. La vegetación del páramo del noroeste de la Sierra Nevada de Santa Marta. Pp. 203-266 in T. Van der Hammen, J. O. Rangel-Ch and A. M. Cleef (eds.). La Sierra Nevada de Santa Marta (Colombia) transecto Buritaca-La Cumbre. Estudios de Ecosistemas Tropandinos. J. Cramer, Berlín, Alemania.

Cleef, A. M., O. Rangel, and S. Salamanca. 1983. Reconocimiento de la vegetación de la parte alta del transecto Parque Los Nevados. Pp. 150-173 in T. Van der Hammen, A. P. Preciado and P. Pinto-Escobar (eds.). La Cordillera Central Colombiana. Transecto Parque Los Nevados. Introducción y Datos Iniciales. Estudios de Ecosistemas Tropandinos. J. Cramer, Vaduz, Liechtenstein.

Duchicela, J., S. Navas, and M. C. Segovia. 2006. Comportamiento funcional de la micorriza arbuscular asociada a Polylepis incana en ecosistema alto andinos bajo condiciones naturales, Ecuador. Revista Ciencia 9(2):123-134.

Elith, J., C. H. Graham, R. P. Anderson, M. Dudík, S. Ferrier, A. Guisan, R. J. Hijmans, F. Huettmann, J. R. Leathwick, A. Lehmann, J. Li, L. G. Lohmann, B. A. Loiselle, G. Manion, C. Moritz, M. Nakamura, Y. Nakazawa, J. McC. Overton, A. T. Peterson, S. Phillips, K. S. Richardson, R. Scachetti-Pereira, R. E. Schapire, J. Soberón, S. Williams, M. S. Wisz, 
and N. E. Zimmermann. 2006. Novel methods improve prediction of species' distributions from occurrence data. Ecography 29:129-151.

Elith, J., S. J. Phillips, T. Hastie, M. Dudík, Y. E. Chee, and C. J. Yates. 2011. A statistical explanation of MaxEnt for ecologists. Divers Distrib 17:43-57.

Fandiño, Y., and D. Caro-Sabogal. 2009. Mapa general de identificación de áreas potenciales de bosques de Polylepis en los Andes Colombianos. Conservación Colombiana 10:7-44.

García-Roselló, E., C. Guisande, H. Juergen, P. Pelayo-Villamil, A. Manjarrés-Hernández, L. González-Vilas, J. GonzálezDacosta, A. Vaamonde, and C. Granado-Lorencio. 2014. Using ModestR to download, import and clean species distribution records. Methods Ecol Evol 5(7):708-713.

Guisan, A., O. Broennimann, R. Engler, M. Vust, N. G. Yoccoz, A. Lehmann, and N. E. Zimmermann. 2006. Using niche-based models to improve the sampling of rare species. Conserv Biol 20:501-511.

Hernández, P. A., I. Franke, S. K. Herzog, V. Pacheco, L. Paniagua, H. L. Quintana, A. Soto, J. J. Swenson, C. Tovar, T. H. Valqui, J. Vargas, and B. E. Young. 2008. Predicting species distributions in poorly-studied landscapes. Biodivers Conserv 17:1353-1366.

Hijmans, R. J., S. E. Cameron, J. L. Parra, P. G. Jones, and A. Jarvis. 2005. Very high resolution interpolated climate surfaces for global land areas. Int J Climatol 25:1965-1978.

Hu, J., and Z. Jiang. 2010. Predicting the potential distribution of the endangered Przewalski's gazelle. J Zool 282: 54-63.

Hueck, K. 1960. Los bosques de Polylepis sericea en los Andes Venezolanos. Boletin IFLA 5:1-32.

IAvH. 2012. Cartografía de Páramos de Colombia Esc. 1:100.000. Proyecto: Actualización del Atlas de Páramos de Colombia. Convenio Interadministrativo de Asociación 11-103, Instituto de Investigación de Recursos Biológicos Alexander von Humboldt y Ministerio de Ambiente y Desarrollo Sostenible. Bogotá D.C. Colombia.

IUCN 2017. The IUCN Red List of Threatened Species. Version 2017-2. URL: www.iucnredlist.org. Acceso: 14 de septiembre de 2017.

Jarvis, A., H. I. Reuter, A. Nelson, and E. Guevara. 2008. Hole-filled SRTM for the globe Version 4, available from the CGIAR-CSI SRTM 90m Database. URL: srtm.csi.cgiar.org.

Kerr, M. S. 2004. A phylogenetic and biogeographic analysis of Sanguisorbeae (Rosaceae), with emphasis on the pleistocene radiation of the high Andean genus Polylepis. Doctoral thesis. Faculty of the Graduate School of the University of Maryland, College Park. USA. Pp. 202.

Kessler, M., and P. Driesch. 1993. Causas e historia de la destrucción de bosques altoandinos en Bolivia. Ecología en Bolivia 21:1-18.

Kumar, S., and T. J. Stohlgren. 2009. Maxent modeling for predicting suitable habitat for threatened and endangered tree Canacomyrica monticola in New Caledonia. J Ecol Nat Environ 1:094-098.

León, V. L. 1991. Estudio de la vegetación vascular de tres bosques de Polylepis sericea Wedd. ubicados en la Sierra Nevada de Mérida. Trabajo Especial de Grado. Departamento de Biología, Facultad de Ciencias. Universidad de Los Andes. Mérida, Venezuela.

Liu, C., P. M. Berry, T. P. Dawson, and R. G. Pearson. 2005. Selecting thresholds of occurrence in the prediction of species distributions. Ecography 28:385-393.

López-Gallego, C. 2015. Monitoreo de poblaciones de plantas para conservación: recomendaciones para implementar planes de monitoreo para especies de plantas de interés en conservación. Instituto de Investigación de Recursos Biológicos Alexander von Humboldt (IAvH). Bogotá D. C., Colombia.

Meneses, L. A., and Y. Herrera-Martínez. 2013. Estudio preliminar de la avifauna asociada a parches de Polylepis quadrijuga (Rosaceae) del páramo de la Rusia, Duitama (Boyacá - Colombia). Revista Luna Azul 36:40-54.

Mommersteeg, H. 1998. Vegetation development and cyclic and abrupt climatic change during the Late Quaternary. Tesis doctoral. University of Amsterdam. Pp. 191+apéndices.

Myers, R. M. 1990. Classical and Modern Regression with Applications. 2. ed., Boston. Duxbury Press.

Padonou, E. A., O. Teka, Y. Bachmann, M. Schmidt, A. M. Lykke, and B. Sinsin. 2015. Using species distribution models to select species resistant to climate change for ecological restoration of Bowé in West Africa. Afr J Ecol 53:83-92.

Papeş, M., and P. Gaubert. 2007. Modelling ecological niches from low numbers of occurrences: assessment of the conservation status of poorly known viverrids (Mammalia, Carnivora) across two continents: Ecological niche modelling of poorly known viverrids. Divers Distrib 13:890-902.

Pearce, J. L., and M. S. Boyce. 2006. Modelling distribution and abundance with presence-only data. J Appl Ecol 43: 405-412.

Pérez, J. A. 2017. Polylepis. En: R. Bernal, S. R. Gradstein and M. Celis (eds.). 2015. Catálogo de plantas y líquenes de Colombia. Instituto de Ciencias Naturales, Universidad Nacional de Colombia, Bogotá. URL: catalogoplantasdecolo mbia.unal.edu.co

Phillips, S. J., R. P. Anderson, and R. E. Schapire. 2006. Maximum entropy modeling of species geographic distributions. Ecol Model 190(3-4):231-259.

Rada, F., C. García-Núñez, and S. Rangel. 2011. Microclimate and regeneration patterns of Polylepis sericea in a treeline forest of the Venezuelan Andes. Ecotropicos 24(1):113-122.

Rada, F., G. Goldstein, A. Azocar, and F. Meinzer. 1985. Daily and seasonal osmotic changes in a tropical treeline species. J Exp Bot 36(167):989-1000.

Ramos, C., S. P. Buitrago, K. L. Pulido, and L. J. Vanegas. 2013. Variabilidad ambiental y respuestas fisiológicas de Polylepis quadrijuga (Rosaceae) en un ambiente fragmentado en el Páramo de la Rusia (Colombia). Rev Biol Trop 
61(1):351-361.

Rangel-Ch, J. O., and H. Arellano-P.2010. Bosques de Polylepis: un tipo de vegetación condenado a la extinción. Pp. 443-478 in J. O. Rangel-Ch (ed.). Colombia Diversidad Biótica X. Cambio Global (Natural) y Climático (Antrópico) en el páramo colombiano. Instituto de Ciencias Naturales-Facultad de Ciencias-Universidad Nacional de Colombia.

Rangel-Ch., J. O., A. M. Cleef, T. Van der Hammen, and R. Jaramillo.1982. Tipos de vegetación en el transecto BuritacaLa Cumbre, Sierra Nevada de Santa Marta (entre 0 y 4100 m). Colomb Geogr 10:1-18.

Rangel-Ch., J. O., and R. Jaramillo.1984. Lista comentada del material herborizado en el transecto Buritaca-La Cumbre (Sierra Nevada de Santa Marta). Pp. 155-176 in T. Van der Hammen, J. O. Rangel-Ch and A. M. Cleef (eds.). La Sierra Nevada de Santa Marta (Colombia) transecto Buritaca-La Cumbre. Estudios de Ecosistemas Tropandinos.

Rangel-Ch., J. O., D. Sánchez, and C. Ariza. 1999. Fitosociología del páramo de Frontino. Pp. 110-120 in C. A. VelaìsquezR., L. N. Parra, D. Sánchez, J. O. Rangel-Ch., C. Ariza and A. Jaramillo (eds.). Tardiglacial y holoceno del norte de la Cordillera Occidental de Colombia. Universidad Nacional de Colombia-COLCIENCIAS. Medellín. Colombia.

Rangel-Ch., J. O., S. P. Díaz, R. M. Jaramillo, and S. V. Salamanca. 1983. Lista del material herborizado en el transecto del Parque Los Nevados (Pteridophyta-Spermatophyta). Pp. 174-205 in T. Van der Hammen, A. P. Preciado and P. Pinto-Escobar (eds.). La Cordillera Central Colombiana. Transecto Parque Los Nevados. Introducción y Datos Iniciales. J. Cramer, Vaduz, Liechtenstein.

Sarmiento, C., C. Cadena, M. Sarmiento, J. Zapata, and O. León. 2013. Aportes a la conservación estratégica de los páramos de Colombia: Actualización de la cartografía de los complejos de páramo a escala 1:100.000. Instituto de Investigación de Recursos Biológicos Alexander von Humboldt. Bogotá, Colombia.

Segovia, M. C. 2014. New insights into the evolutionary history of the complex Andean genus Polylepis (Rosaceae: Sanguisorbeae) and implications for conservation and management. Tesis doctoral. University of Florida, USA. Pp. 182.

Soberoìn, J. 2010. Niche and area of distribution modeling: a population ecology perspective. Ecography 33:159-167.

Soberoìn, J., and M. Nakamura. 2009. Niches and distributional areas: Concepts, methods, and assumptions. Proc Natl Acad Sci USA 106:19644-19650.

Squeo, F. A, F. Rada, A. Azocar, and G. Goldstein. 1991. Freezing tolerance and avoidance in high tropical Andean plants: Is it equally represented in species with different plant height? Oecologia 86:378-382.

Tarifa, T., and E. Yensen.2001. Mamíferos de los bosques de Polylepis de Bolivia. Rev Bol Ecol 9:29- 44.

Thorn, J. S., V. Nijman, D. Smith, and K. A. I. Nekaris. 2009. Ecological niche modelling as a technique for assessing threats and setting conservation priorities for Asian slow lorises (Primates: Nycticebus). Divers Distrib 15:289-298.

Toranza, C., A. Brazeiro, and R. Maneyro. 2016. Anfibios amenazados de Uruguay: efectividad de las áreas protegidas ante el cambio climático. Ecología Austral 26:138-149.

Torres, V. 2006. Pliocene-pleistocene evolution of flora, vegetation and climate: a palynological and sedimentological study of a 586-m core from the Bogotaì basin, Colombia. University of Amsterdam, IBED. Pp. 181.

Valderrama, S. V., and J. C. Verhelst. 2009. Avifauna asociada a los bosques de Polylepis en Colombia. Conservación Colombiana 10:45-68.

Van der Hammen, T. 1986. La Sabana de Bogotá y su lago en el Pleniglacial Medio. Caldasia 15:249-262.

Van der Hammen, T., and H. Hooghiemstra. 1997. Chronostratigraphy and correlation of the Pliocene and Quaternary of Colombia. Quatern Int 40:81-91.

Van der Hammen, T., J. H. Werner, and H. van Dommelen. 1973. Palynological record of the upheaval of the northern Andes: A study of the Pliocene and lower Quaternary of the Colombian Eastern Cordillera and the early evolution of its high Andean biota. Rev Palaeobot Palyno 16:1-122.

Vargas, J. O. 2002. Disturbios, patrones sucesionales y grupos funcionales de especie en la interpretación de matrices de paisaje en los páramos.

Vargas, J. O. 2013. Disturbios en los páramos andinos. Pp. 39-57 in J. Cortés-Duque and C. E. Sarmiento Pinzón. Visión socioecosistémica de los páramos y la alta montaña colombiana, Memorias del proceso de definición de criterios para la delimitación de páramos. Instituto de Investigación de Recursos Biológicos Alexander von Humboldt.

Vásquez, E., B. Ladd, and N. Borchard. 2014. Carbon storage in a high-altitude Polylepis woodland in the Peruvian Andes. Alpine Bot 124(1):71-75.

Vélez, V., J. Cavelier, and B. Devia. 1998. Ecological traits of the tropical treeline species Polylepis quadrijuga (Rosaceae) in the Andes of Colombia. J Trop Ecol 15:771-787.

Verweij, P. A. 1995. Spatial and temporal modelling of vegetation patterns-burning and grazing in the paramo of Los Nevados National Park, Colombia. Tesis doctoral. Faculty of Science (FNWI), Institute for Biodiversity and Ecosystem Dynamics (IBED). University of Amsterdam.

Zutta, B. R., P. W. Rundel, S. Saatchi, J. D. Casana, P. Gauthier, A. Soto, Y. Velazco, and W. Buermann. 2012. Prediciendo la distribución de Polylepis: bosques Andinos vulnerables y cada vez más importantes. Rev Per Biol 19(2):205-212. 